\title{
Surgical treatment of benign endobronchial tumours
}

\author{
P HALTTUNEN, H MEURALA, C-G STANDERTSKJÖLD-NORDENSTAM
}

From the Department of Thoracic and Cardiovascular Surgery and the Department of Radiology, Helsinki University Central Hospital, Helsinki, Finland

ABSTRACT Four cases of benign endobronchial tumour are reported which were successfully treated by bronchial resection. In two cases (of fibroma and leiomyoma respectively) a cylinder of bronchus alone was resected; in one case (lipoma) a healthy right upper lobe was preserved by a bronchoplastic procedure and in the other (chondroma) the tumour was removed with the right lower lobe, which was irreversibly damaged. It is important to recognise that such tumours are unsuitable for treatment by endoscopic means alone.

Benign endobronchial tumours are rare. We have found reports of 21 fibromas, ${ }^{1} 20$ leiomyomas, ${ }^{2} 73$ lipomas, ${ }^{3}$ and only six chondromas. ${ }^{4}$ This paper concerns one fibroma, one leiomyoma, one lipoma, and one chondroma, which were treated by local bronchial resection with or without a bronchoplastic procedure in three cases and by lobectomy in one case. We wish to emphasise that certain types of endobronchial tumour are not suitable for treatment by endoscopic means alone.

\section{Case 1 (fibroma)}

A 32-year-old man had two episodes of bronchitis in 1969. In 1974 he visited a doctor because of pain in the left chest, which had been increasing in severity for two years. A chest radiograph showed an appreciable decrease in the volume of the left lung, with mediastinal shift to the left but without any other signs of atelectasis. The results of spirometry were normal. An isotope perfusion scan showed hardly any perfusion of the left lung.

Bronchoscopy showed a smooth pink tumour filling the left main bronchus and protruding into the trachea. Biopsy of the tumour did not give any specific diagnosis. Sputum cytology was normal. At thoracotomy the left main bronchus and the tracheal bifureation were exposed and surrounded by tapes. The main bronchus was incised immediately distal to the bifurcation, and a tumour $3 \mathrm{~cm}$ in diameter was found protruding from the bronchus (fig 1). The tumour was connected to the medial bronchial wall by a stalk that was $0.5 \mathrm{~cm}$ thick. A cylinder of bron-

Address for reprint requests: Dr $P$ Halttunen, Department of Thoracic and Cardiovascular Surgery, Helsinki University Central Hospital, Haartmaninkatu 4, SF-00290 Helsinki 29, Finland. chus less than $1 \mathrm{~cm}$ in length was resected, leaving normal bronchial wall on either side of the stalk. The bronchial ends were then anastomosed end to end with continuous atraumatic $2-0$ chromic catgut. Macroscopically, the stalk of the tumour originated from a cartilaginous ring. Histologically, the tumour was covered by cylindrical epithelium with local metaplasia. The stroma consisted of collagen fibres and fibroblasts, local bronchial glands and blood vessels, and some cystic spaces lined by epithelium-compatible with a fibroma.

The patient's recovery was uneventful. Seventeen months after the operation the chest radiograph was normal and bronchoscopy showed only slight narrowing at the site of the anastomosis. An isotope scan showed moderate perfusion of the left lung much better than before operation but not as good as in the right lung. The patient has since moved abroad.

\section{Case 2 (leiomyoma)}

A 28-year-old man had suffered from dyspnoea and dry cough since May 1974 . Since then the chest radiograph had showed a progressive shrinking of the left lung and a corresponding shift of the mediastinum to the left. Previous chest photofluorograms, the latest of which was done in 1972, had been normal.

At bronchoscopy a smooth spherical tumour with a broad base was seen near the orifice of the left main bronchus, which was almost occluded. Histological examination showed it to be a benign leiomyoma. At operation in July 1974 via a left thoracotomy, a $2 \cdot 5-\mathrm{cm}$ segment of the left main bronchus was resected (fig 2 ). In this specimen a smooth tumour about $2 \mathrm{~cm}$ in diameter was seen 


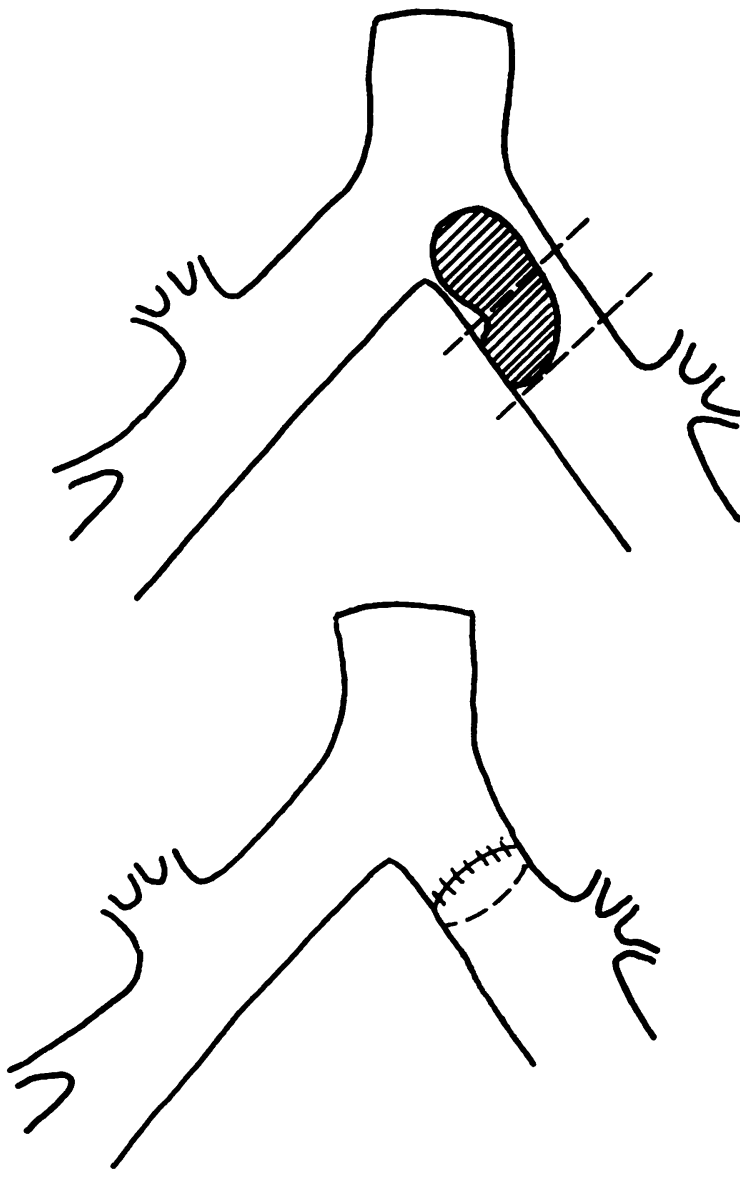

Fig 1 Schematic drawing of endobronchial fibroma (case 1) treated by local bronchial resection.

originating from the pars membranacea. Bronchial anastomosis was performed with 2-0 atraumatic chromic catgut and the suture line was covered with a flap of the parietal pleura. The histological diagnosis of leiomyoma was confirmed.

Three months later the patient returned for follow-up. His chest radiograph was no different from the preoperative ones. Spirometry showed the vital capacity (VC) to be 4.801 ( $85 \%$ of normal), FEV , 3.82 1 (83\% of normal), and FEV,/VC $80 \%$ (normal 81), whereas before operation the figures were VC 3.851 (75\% of normal), FEV 2.651 (63\% of normal), and $\mathrm{FEV}_{1} / \mathrm{VC} 69 \%$. On radiospirometry, which was done only after operation, the distribution between the right and the left lung was $74 \% / 26 \%$, and the vital capacity $77 \% / 23 \%$. Bronchoscopy showed the left main bronchus to be patent, with only slight narrowing dorsally at the site
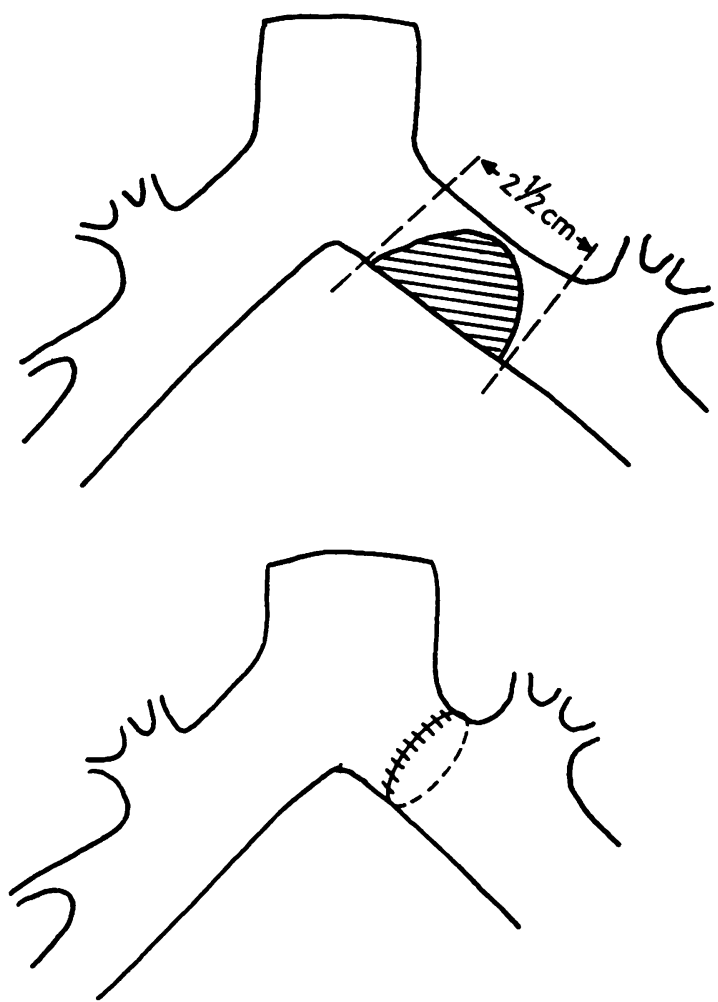

Fig 2 Schematic drawing of endobronchial leiomyoma (case 2) treated by resecting $2.5 \mathrm{~cm}$ of the left main bronchus.

of anastomosis. At subsequent follow-ups the patient has been free of symptoms.

\section{Case 3 (lipoma)}

A 40-year-old woman had suffered from right-sided pneumonia in 1971 and 1972 and several episodes of febrile bronchitis during later years. In 1978 an increasing amount of mucous expectoration and wheezy breathing appeared, but no dyspnoea. From late 1979 she was so tired that she had to resign from her job. During the spring of 1980 she had three attacks of febrile bronchitis. At that time the chest radiograph (fig $3 a$ ) showed atelectasis of the right middle and lower lobes. Spirometry showed a VC of 1.971 (50\% of normal), FEV 1.261 (40\% of normal), and $\mathrm{FEV}, \mathrm{VC} 78 \%$ (normal $82 \%$ ). Bronchoscopy in June showed a smooth tumour covered by mucosa occluding the right main bronchus, extending beyond the carina, and slightly narrowing the left main bronchus as well. Histological examination showed only fibrosis and chronic inflammation. The chest radiograph now also 


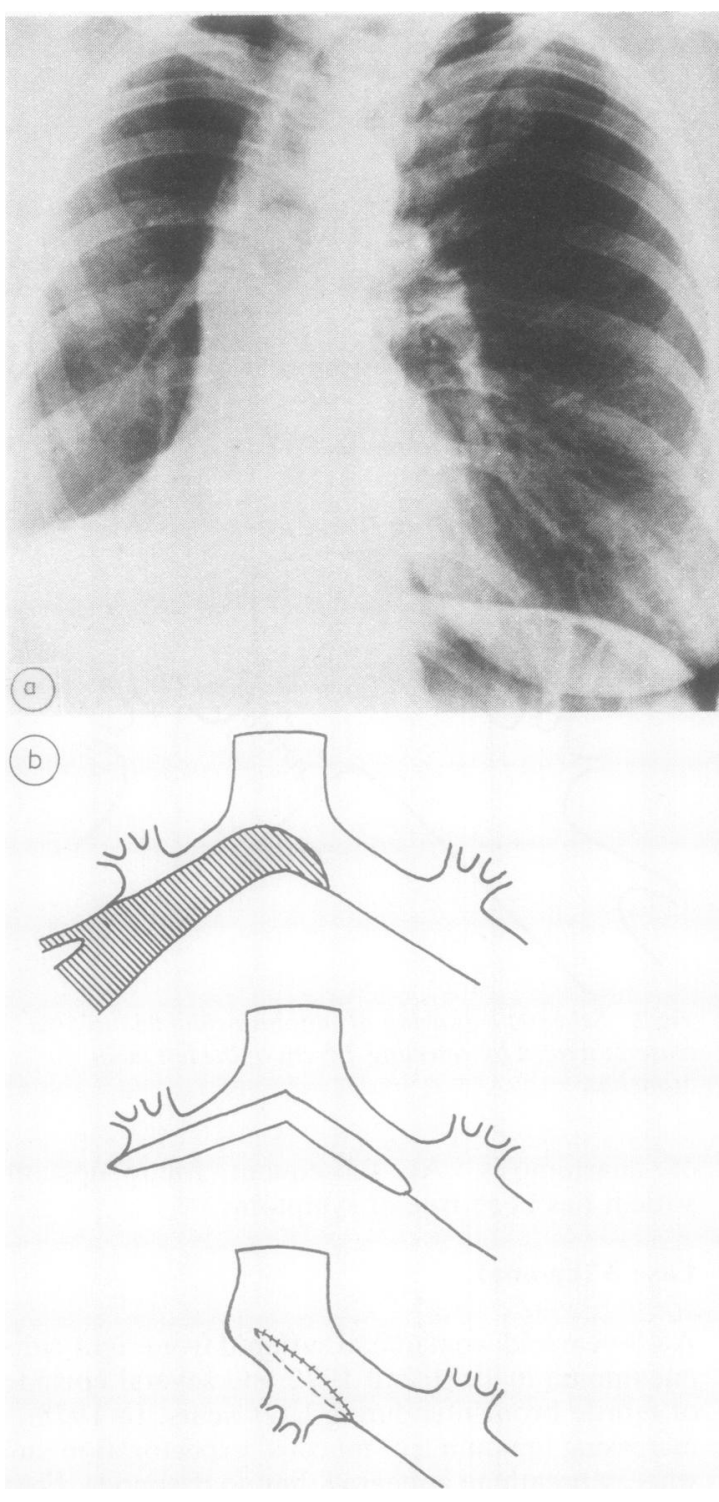

showed mediastinal shift to the right. In 1971 chest photofluorograms had shown atelectasis of the left lower lobe, but no mediastinal shift.

Right thoracotomy showed atelectasis of the right middle and lower lobes and the corresponding lobar bronchi were dissected. The azygos vein was ligated and transected. The main bronchus was then resected, the upper-lobe bronchus and lateral flap of the main bronchus being saved for use in reconstruction (fig $3 b)$. The resection was extended to the carina so that the tumour could be totally exstirpated with a narrow margin of normal tissue. The left main bron-

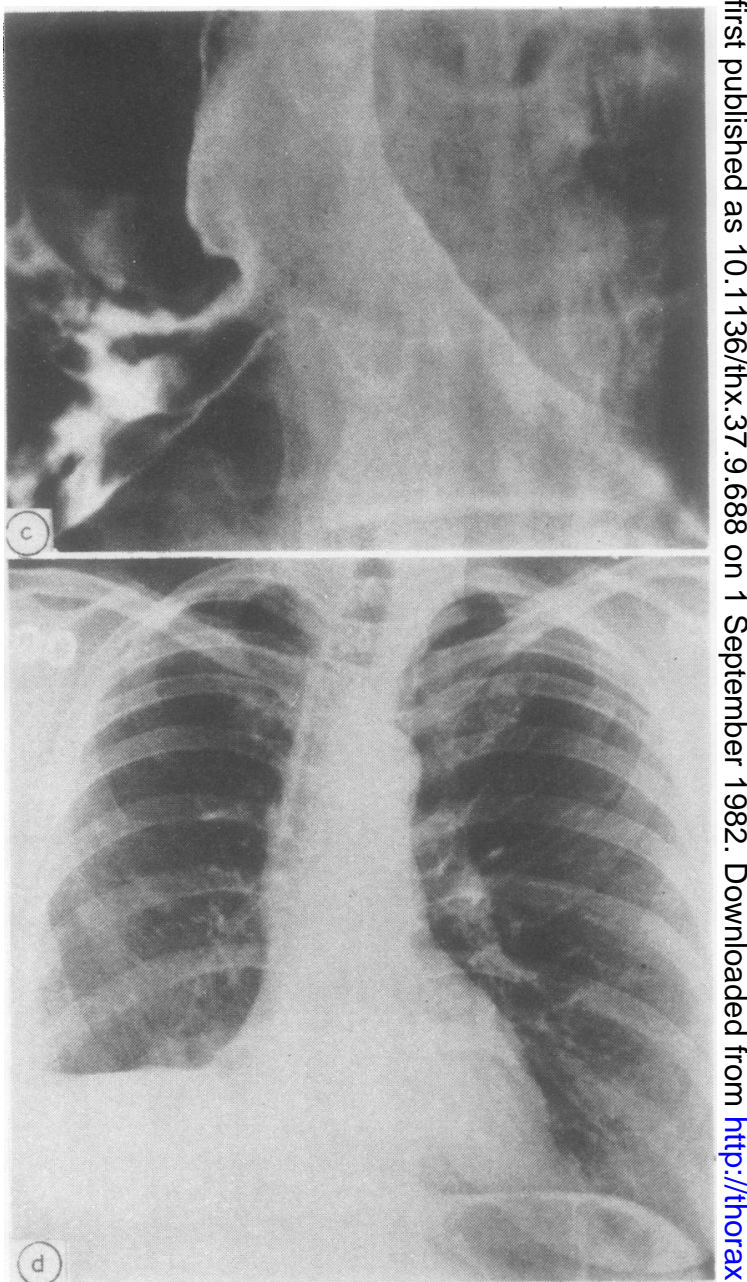

Fig 3 Case 3: (a) Preoperative chest radiograph showing total atelectasis of the right middle and lower lobes, the upper lobe filling almost the entire right pleural cavity and the mediastinum shifted to the right. (b) Schematic drawing of endobronchial lipoma treated by resection of the middle 0 and lower lobes, the upper lobe being saved by the use of a bronchoplastic technique. (c) Tracheobronchogram three months after operation showing the result of the bronchoplasty. (d) Chest radiograph three months after operation.

chus had to be temporarily intubated via the thoracotomy. A plastic transverse closure of the bronchial aperture was then performed with a con tinuous 3-0 atraumatic polyglycolic acid suture.

The patient's recovery was uneventful. Cinetracheobronchography (fig $3 c$ ) two weeks after oper $\frac{0}{6}$ ation showed the right upper-lobe bronchus to be fully open. Three months later spirometry showed? the VC to be 3.131 (80\% of normal), FEV, $2.15 \mathbb{Q}$ 
(68\% of normal), and $\mathrm{FEV}_{1} / \mathrm{VC} 69 \%$ (normal $82 \%$ ). Bronchoscopy indicated that the carina and the anastomosis had healed well and that the right upper-lobe bronchus was patent. The chest radiograph showed the remaining right lung filling the corresponding pleural cavity with only slight mediastinal shift (fig $3 d$ ). The patient was free of symptoms.

\section{Case 4 (chondroma)}

A 54-year-old man had suffered from recurrent episodes of bronchitis since 1965. This led to further investigations in January 1972. The chest radiograph showed some pleural adhesions on the right (he had had an exudative pleuritis on that side at the age of 10). Spirometry and bronchoscopy gave normal results.

In September 1974 the patient was examined again because of two episodes of haemoptysis. The chest radiograph did not differ from that of 1972 . Bronchoscopy showed a smooth intrabronchial tumour occluding the right posterobasal segmental bronchus. A biopsy of the tumour did not provide a specific diagnosis. On spirometry the VC was 3.91 (91\% of normal), FEV 2.41 (75\% of normal), and $\mathrm{FEV}_{1} / \mathrm{VC} 72 \%$ (normal $77 \%$ ).

At thoracotomy the right lung was seen to have adhesions from the earlier pleuritis. The tumour was located in the bronchus of the right lower lobe. The entire right lower lobe had to be resected because of the peripheral location of the tumour (fig 4). In the specimen the orifice of the posterobasal segment was occluded by an intrabronchial, smooth, pale tumour $1 \mathrm{~cm}$ in diameter. Histologically this was composed of cartilaginous islands separated by fat and coated with normal bronchial cylindrical epithelium - that is, it was a bronchial chondroma. The patient was examined regularly until April 1981. No pertinent pathological findings emerged from chest radiography. He experienced some dyspnoea on exercise but was greatly overweight and also suffered from cardiac insufficiency.

\section{Discussion}

Removal of a benign endobronchial tumour through the bronchoscope is indicated when the tumour is small or pedunculated, ${ }^{35}$ though in some cases it may cause profuse bleeding or perforation of the bronchus. If, however, the tumour occludes the bronchus irreversible peripheral changes in the lung may have occurred (as in case 3), and in such cases neither a bronchoscopic examination nor bronchography may give sufficient information about the bronchial tree distal to the tumour. Moreover, a histological diag-
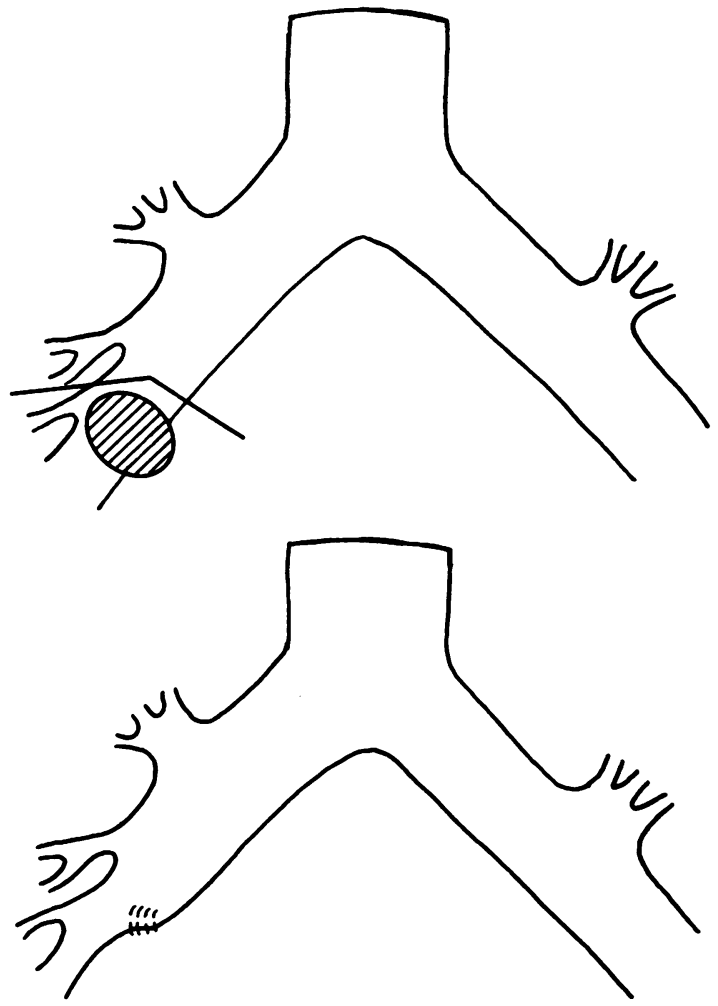

Fig 4 Schematic drawing of endobronchial chondroma (case 4) treated by right lower lobectomy.

nosis of endobronchial tumour may not have been obtained (cases 1, 3, and 4). In these cases endobronchial treatment is not advisable and operative evaluation is required. Furthermore, some of the benign tumours may later recur or undergo malignant degeneration (for example, chondroma ${ }^{4}$ and leiomyoma ${ }^{2}$ ) and should therefore be treated radically by bronchial resection. We therefore think that local resection of the bronchus is the method of choice for the treatment of sessile or occluding benign endobronchial tumours in cases without irreversible changes in the peripheral lung. ${ }^{67}$

If there is damage to the lung combined with the tumour a lobectomy or segmentectomy is necessary. The value of saving as much as possible of the healthy lung must be borne in mind, and this can be achieved by local bronchial resection or a bronchoplastic operation.

\section{References}

' Corona FE, Okeson GC. Endobronchial fibroma. Am Rev Respir Dis 1974;110:350-3.

${ }^{2}$ Shahian DM, McEnany MT. Complete endobronchial 
excision of leiomyoma of the bronchus. J Thorac Cardiovasc Surg 1979;77:87-91.

${ }^{3}$ Medelli J, Abet D, Bertoux JP, Vermynck JP, Giroulle H, Pietri J, Goudot B. Le lipome bronchique. A propos de quatre observations. Ann Chir 1980;34:327-31.

4 Brewster DC, MacMillan IKR. Chondroma of the trachea. Ann Thorac Surg 1975;19:576-84.
${ }^{5}$ Lundgren R, Winblad B. Resection of bronchial chondroma through the flexible fiberoptic bronchoscope. Endoscopy 1980;12:81-2.

- Farsad GRH, Makoui C. Endobronchial lipoma. Am Surg 1981;47:236-8.

' Laustela E, Koskinen R, Ahlqvist J. Leiomyoma of the bronchus. Annales Chirurgiae et Gynaecologiae Fenniae 1974;63:346-9. 